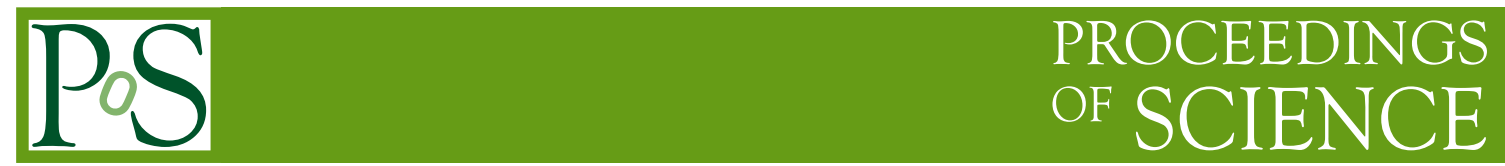

\title{
Mesons from generalizations of AdS/CFT
}

\author{
Johanna Erdmenger* \\ Max-Planck-Institut für Physik (Werner-Heisenberg-Institut), Föhringer Ring 6, 80805 \\ München, Germany \\ E-mail: jke@mppmu.mpg.de
}

We give a brief overview over AdS/CFT and discuss generalizations to field theories with running gauge coupling, partially or fully broken supersymmetry and with added flavor degrees of freedom. Moreover we discuss applications such as chiral symmetry breaking and meson spectra.

LIGHT CONE 2008 Relativistic Nuclear and Particle Physics

July 7-11 2008

Mulhouse, France

${ }^{*}$ Speaker. 


\section{Introduction}

String theory originated as a theory of hadrons in the 1960's, when it was noticed that hadron spectra coincide with excited states of a rotating string. String theory as a theory of strong interactions was abandoned however since four-dimensional string theory contains tachyonic modes. From the beginning of the 1970's, Quantum Chromodynamics (QCD) has established itself as a very successful quantum field theory of strong interactions, which is by now very well tested experimentally. String theory took a rather different route due to the fact that it contains a graviton in its spectrum, and is by now a very promising candidate for a unified quantum theory of all four fundamental interactions.

Within the last ten years, following the paper by Maldacena [1] introducing the AdS/CFT correspondence, a wealth of interesting new relations between modern string theory and quantum field theory have been found. In this review we discuss in particular generalizations of the original AdS/CFT correspondence to gauge/gravity dualities for confining gauge theories with flavor degrees of freedom.

\section{Gauge/gravity duality}

\subsection{AdS/CFT correspondence}

The AdS/CFT correspondence is a duality which - in its simplest form - maps a quantum field theory at strong coupling to a gravity theory at weak coupling. The best known example is the map between $\mathscr{N}=4$ supersymmetric $U(N)$ Yang-Mills theory, which is mapped to type IIB supergravity on the space $A d S_{5} \times S^{5}$. Here $A d S_{5}$ denotes five-dimensional Anti-de Sitter space and $S^{5}$ denotes the five-sphere. Anti-de Sitter space is a space of constant negative curvature which has a boundary. The metric of $A d S_{5} \times S^{5}$ may be written in the form

$$
d s^{2}=L^{2}\left(\frac{1}{u^{2}} \eta_{i j} d x^{i} d x^{j}+\frac{d u^{2}}{u^{2}}+d \Omega_{5}^{2}\right),
$$

where $L$ is the AdS radius and $\eta_{i j}$ is the standard 3+1-dimensional Minkowski metric. There is a boundary of $A d S_{5}$ at $u=0$. It is sometimes convenient to perform a coordinate transformation and to write the metric of $A d S_{5}$ in the form

$$
d s^{2}=e^{2 r / L} \eta_{i j} d x^{i} d x^{j}+d r^{2}
$$

The boundary is then located at $r \rightarrow \infty$.

The AdS/CFT correspondence arises from string theory in a particular low-energy limit in which the 't Hooft coupling is large and fixed, while $N \rightarrow \infty$, such that the planar limit of the gauge theory is considered. $\mathscr{N}=4$ Super Yang-Mills theory is a conformal field theory in which the beta function vanishes to all orders in perturbation theory. Therefore it has a $S O(4,2)$ conformal symmetry, which coincides exactly with the isometry of $A d S_{5}$. Similarly, the $S U(4) \simeq S O(6) \mathrm{R}$ symmetry of the field theory coincides with the isometry of the five-sphere $S^{5}$.

The AdS/CFT correspondence has been developed further in [2] where a field-operator map has been established: There is a one-to-one correspondence between gauge-invariant operators in 
the field theory and supergravity fields on $A d S_{5}$. This maps gauge invariant operators of $\mathscr{N}=$ 4 Yang-Mills theory in a particular irreducible representation of $S U(4)$ to supergravity fields in the same representation. These five-dimensional supergravity fields are obtained by Kaluza-Klein reduction of the original ten-dimensional supergravity fields on the five-sphere $S^{5}$. There is a precise relation between the Kaluza-Klein mass $m_{\text {sugra }}$ of the supergravity fields and the dimension $\Delta$ of the dual operator. For scalars this relation is $m_{\text {sugra }}^{2}=\Delta(\Delta-d)$, with $d$ the dimension of the AdS boundary. For our purposes, $d=4$. The asymptotic behaviour of the supergravity fields at the AdS boundary is of central importance. For a given supergravity field $\phi$ of Kaluza-Klein mass $m$ it is given by

$$
\phi(u) \sim u^{d-\Delta} \phi_{0}+u^{\Delta}\langle\mathscr{O}\rangle
$$

for $u \rightarrow 0$. As discussed in [2], the boundary value $\phi_{0}$ may be identified with the source of the gauge theory-operator $\mathscr{O}$, and $\langle\mathscr{O}\rangle$ is the VEV of $\mathscr{O}$. - The AdS/CFT correspondence has been tested in numerous examples, among which the calculation of correlation functions and of the conformal anomaly [3].

The string-theoretical origin of the AdS/CFT correspondence arises from the two different interpretations of D3 branes, i. e. 3+1-dimensional hyperplanes within 9+1-dimensional space. On the one hand, D3 branes are hyperplanes on which open strings can end. In the low-energy limit where only massless string excitations are taken into account, the degrees of freedom on a stack of $N$ D3 branes correspond to $\mathscr{N}=4 U(N)$ Super Yang-Mills theory in four-dimensional Minkowski space. On the other hand, D3 branes are a solitonic solution of ten-dimensional IIB supergravity. As such they are massive extended objects which curve the space around them. In the near-horizon limit, which is also a low-energy limit, this curved space is just $A d S_{5} \times S^{5}$. The excitations in this curved-space background are closed strings whose massless mode corresponds to gravitons. In the Maldacena limit in which the 't Hooft coupling is large and fixed, while $N \rightarrow \infty$, the string modes decouple, such that only supergravity, i.e. pointlike particles, survive.

\subsection{Generalizations of AdS/CFT}

It is an appealing idea to generalize this gauge/gravity duality to less symmetric quantum field theories which at least in some respects are similar to QCD. A number of avenues have been pursued over the last few years. These are listed in the following:

- Holographic RG flows: By considering more involved metrics than $A d S_{5} \times S^{5}$ with a reduced degree of symmetry, it is possible to construct gravity duals of field theories with running gauge coupling. Important examples are [4].

The fifth dimension - perpendicular to the boundary on which the field theory lives - may be interpreted as an energy scale. Some of these holographic renormalization group flows flow to confining field theories in the infrared, as may be shown by calculating the Wilson loop within the dual gravity theory, which follows an area law in this case.

- Adding flavor: In $\mathscr{N}=4$ theory, all fields are in the adjoint representation of the gauge group, since all fields are in the same supermultiplet as the gauge field. Matter in the fundamental representation of the gauge group, i.e. quarks, may be added by adding further D-branes to the original stack of $N$ D3 branes. The prototype example is the addition of 
D7 brane probes. This corresponds to adding $\mathscr{N}=2$ hypermultiplets in the fundamental representation of the gauge group. By combining the addition of brane probes with the deformation of the gravity background, a gravity dual description of spontaneous chiral symmetry breaking by a quark condensate is obtained, as well as meson spectra involving Goldstone bosons. An alternative approach which provides a gravity dual realization of $U\left(N_{f}\right) \times U\left(N_{f}\right) \rightarrow U\left(N_{f}\right)$ chiral symmetry breaking uses D4, D8 and D8 branes. A review on mesons in gauge/gravity duals may be found in reference [5].

- AdS/QCD: Whereas this review is devoted to the 'top-down' approach where string-theory models are developed fo describing field theory features, it should also be mentioned that there are important efforts in a 'bottom-up' approach of constructing phenomenological fivedimensional models for describing QCD phenomenology, for instance [6, 7]. These models achieve considerable agreement with experimental results for masses and decay constants in QCD. For applications in the context of the light cone, see the contribution of S. Brodsky to these proceedings.

- Hard scattering: Hard scattering of glueballs may be mapped to string amplitudes in $A d S_{5} \times$ $S^{5}$. This provides in particular a unified description of the soft and hard pomeron [8]. Recently, a classical string configuration was proposed for gluon scattering itself [9].

\section{Flavor and Chiral symmetry breaking}

\subsection{Adding Flavor}

The original AdS/CFT correspondence only involves fields in the adjoint representation of the gauge group. For generalising the correspondence to quark degrees of freedom, which are in the fundamental representation of the gauge group, additional ingredients are necessary. The simplest way to obtain quark bilinear operators within gauge/gravity duality is to add a D7 brane probe [10]. This is done in such a way that the D7 brane probe extends in space-time as given in Table 1, where 0 is the time direction.

\begin{tabular}{|c|c|c|c|c|c|c|c|c|c|c|}
\hline & 0 & 1 & 2 & 3 & 4 & 5 & 6 & 7 & 8 & 9 \\
\hline $\mathrm{D} 3$ & $\mathrm{X}$ & $\mathrm{X}$ & $\mathrm{X}$ & $\mathrm{X}$ & & & & & & \\
\hline $\mathrm{D} 7$ & $\mathrm{X}$ & $\mathrm{X}$ & $\mathrm{X}$ & $\mathrm{X}$ & $\mathrm{X}$ & $\mathrm{X}$ & $\mathrm{X}$ & $\mathrm{X}$ & & \\
\hline
\end{tabular}

Table 1: Embedding of the D7 brane probe into 9+1 dimensional space relatively to the D3 branes.

backreaction of the D7 branes on the geometry.

The field theory corresponding to this brane set-up is a $\mathscr{N}=2$ supersymmetric $U(N)$ gauge theory, which in addition to the degrees of freedom of $\mathscr{N}=4$ Super Yang-Mills contains $N_{f}$ hypermultiplets in the fundamental representation of the gauge group, where $N_{f}$ is given by the number of D7 branes. $N_{f}$ must be small in the probe limit.
The term 'brane probe' refers to the fact that only a very small number of $\mathrm{D} 7$ branes is added, while the number of $\mathrm{D} 3$ branes, $N$, which also determines the rank of the gauge group $U(N)$, goes to infinity. In this limit we neglect the 
On the supergravity side of the duality, the $\mathscr{N}=4$ degrees of freedom are described by supergravity on $A d S_{5} \times S^{5}$ as before. However in addition, there are new degrees of freedom corresponding to the D7

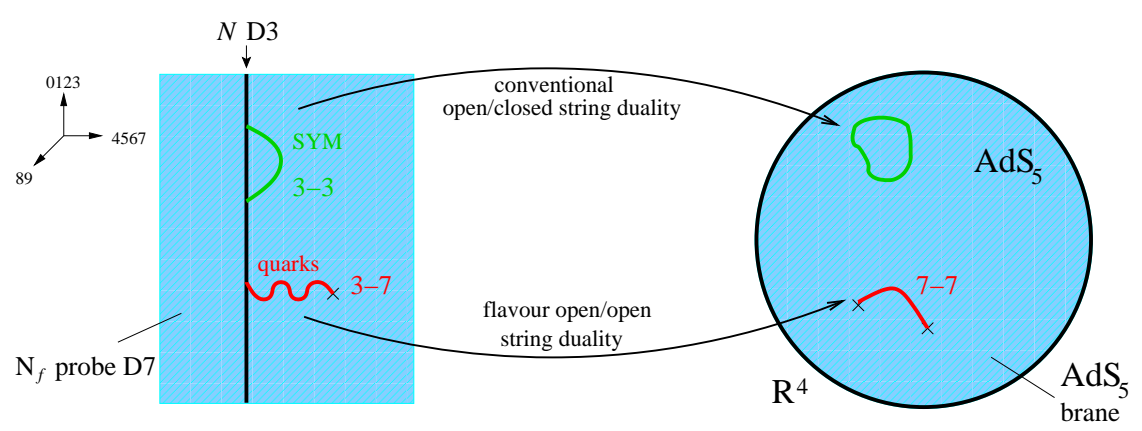

Figure 1: Schematic representation of the AdS/CFT duality with added flavor. In addition to the original AdS/CFT duality, open string degrees of freedom representing quarks are mapped to open strings beginning and ending on the D7 probe wrapping $A d S_{5} \times S^{3}$ inside $A d S_{5} \times S^{5}$. For simplicity, the five-sphere is not drawn in this picture.

brane probe within the ten-dimensional curved space. The low-energy degrees of freedom of this brane are described by the Dirac-Born-Infeld action. These correspond to open string fluctuations on the D7 probe. It turns out that the minimum action configuration for the D7 brane probe corresponds to the probe wrapping an $A d S_{5} \times S^{3}$ subspace of $A d S_{5} \times S^{5}$.

The new duality conjectured in [10] is an open-open string duality, as opposed to the original AdS/CFT correspondence which is an open-closed string duality. The duality states that in addition to the original AdS/CFT duality, gauge invariant field theory operators involving fundamental fields are mapped to fluctuations of the D7 brane probe on $A d S_{5} \times S^{3}$ within $A d S_{5} \times S^{5}$. This is shown in Figure 1.

A particularly interesting feature arises if the $D 7$ brane probe is separated from the stack of D3 branes in either the $x_{8}$ or $x_{9}$ directions, where the indices refer to the coordinates given in Table 1. This corresponds to giving a mass to the fundamental hypermultiplet. In this case the radius of the $S^{3}$ becomes a function of the radial coordinate $r$ in $\mathrm{AdS}_{5}$. At a radial distance from the deep interior of the AdS space given by the hypermultiplet mass, the radius of the $S^{3}$ shrinks to zero. From a five-dimensional AdS point of view, the D7 brane probe seems to 'end' at this value of the AdS radial coordinate.

The scalar mode of the D7 brane probe embedding with dimension $\Delta=3$ (i.e. supergravity mass $m_{\text {sugra }}^{2}=-3$ ) maps to the fermion bilinear $\tilde{\psi} \psi$ in the dual field theory. This mode corresponds to a imaginary AdS mass. However this mass is above the BreitenlohnerFreedman bound [11] for $A d S_{5}\left(m_{\mathrm{BF}}^{2}=-4\right)$ and thus guarantees stability. For this is important that the D7 branes do not carry any net charge from the five-dimensional point of view, since they wrap a topologically trivial cycle with zero flux.

Fluctuations of the D7 brane give rise to

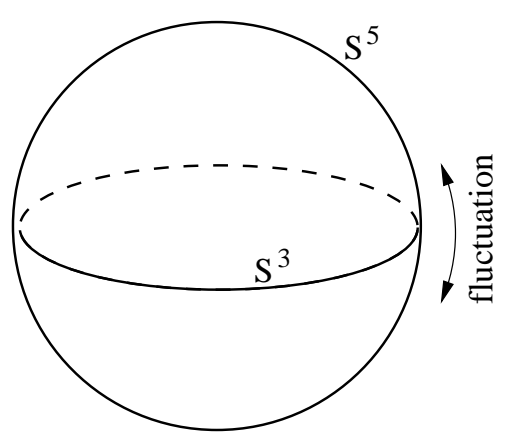

Figure 2: Fluctuations of the $S^{3}$ wrapped by the D7 probe inside $S^{5}$. These modes give rise to the meson masses. 
meson masses [12]. For this, fluctuations of the D7 brane probe of the form $\delta w(\rho, x)=f(\rho) \sin (k$. $x)$ are considered. Here $\rho$ is the radial direction in the four-dimensional space spanned by the cartesian 4, 5, 6,7 directions (see Table 1), and $x$ denote the coordinates on 3+1-dimensional Minkowski space at the boundary of the five-dimensional Anti-de Sitter space. The meson masses are defined by $M^{2}=-k^{2}$ for the wavevector $k$.

\subsection{Chiral symmetry breaking}

To obtain a gravity dual of spontaneous chiral symmetry breaking by a quark condensate [13], the addition of a D7 brane probe is combined with an appropriate deformation of the $A d S_{5} \times S^{5}$ space. A suitable background is the one introduced by Constable-Myers [14]. This background has a single deformation parameter $b$, which may be related to $\Lambda_{\mathrm{QCD}}$ on the field theory side, and a singularity at $r=b$. On the field theory side, the $\mathscr{N}=4$ Super-Yang-Mills theory is deformed to a non-supersymmetric, confining field theory, as can be seen by a Wilson loop analysis.

By adding a D7 brane probe to this background, we obtain a gravity dual of a confining $U(N)$ gauge theory with one flavor [13]. This theory has an $U(1)_{\mathrm{A}}$ axial symmetry. In the $N \rightarrow \infty$ limit, this symmetry is non-anomalous. It can thus be spontaneously broken by the quark condensate $\langle\bar{\psi} \psi\rangle$.

The $U(1)$ rotational invariance in the two space directions perpendicular to the D7 brane (see Table 1) corresponds to the field theory' $\mathrm{s}$ $U(1)_{A}$. The fluctuations in the two directions transverse to the probe are associated to the quark bilinear operator. The UV asymptotic behaviour of the embedding scalars is of the form $|w| \propto m e^{-r}+$ $c e^{-3 r} . m$ and $c$ fix the

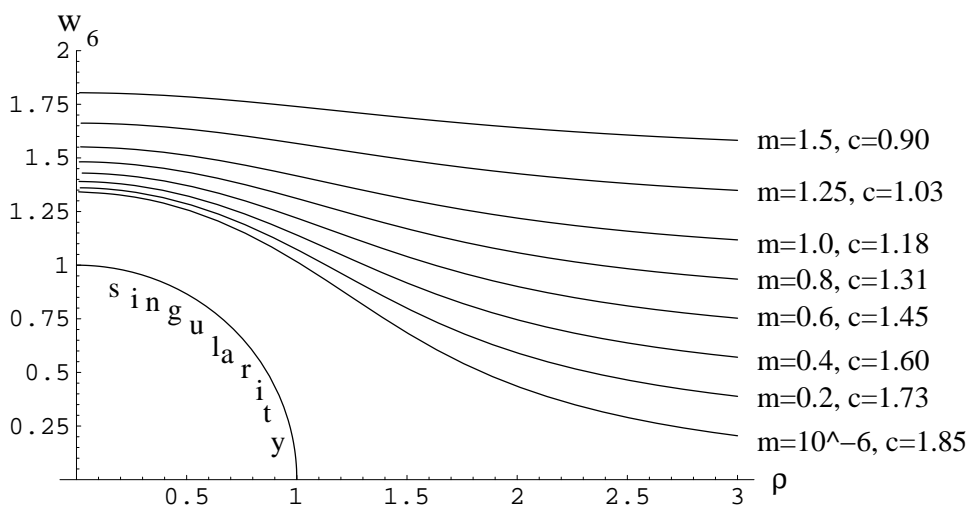

Figure 3: Regular solutions for $\mathrm{D} 7$ embeddings in the Constable-Myers background (Scale $b=1, b \sim \Lambda_{\mathrm{QCD}}$ ). $w_{6}$ denotes one of the coordinates perpendicular to the D7 probe, $\rho$ given by $r^{2}=\rho^{2}+w_{6}{ }^{2}$ is related to the radial coordinate $r$ which corresponds to an energy scale: In the IR $r$ is small and in the UV $r$ is large. The fact that the D7 branes bend corresponds to a geometric realization of spontaneous $U(1)$ symmetry breaking. From [13].

boundary conditions for the second order supergravity equations of motion.

Following the standard AdS/CFT prescription (see Section 2.1 above), we associate the coefficient $m$ with the quark mass and $c$ with the quark condensate $\langle\bar{\psi} \psi\rangle$. Solutions with $m \neq 0$ explicitly breaks the $U(1)_{A}$ symmetry. The solution with $m=0$, but $c \neq 0$ realizes spontaneous breaking of chiral symmetry. Imposing the regularity of the solution in the IR selects a condensate $c$ for each given quark mass $m$. Figure 3 shows regular solutions of the D7-brane equation of motion for different values of $m$. There are three important features of the solutions. The first is the presence of a screening effect: All regular solutions end before reaching the singularity. The presence of 
a condensate $(c \neq 0)$ is essential for this behavior. Moreover we see a geometrical realization of the $U(1)_{A}$ spontaneous breaking, since for $m \rightarrow 0$ we still have $c \neq 0$. Finally, at large $m$ we have $c \sim 1 / m$, as expected from field theory.

Since there is spontaneous symmetry breaking for $m \rightarrow$ 0 , we expect a Goldstone boson in the meson spectrum. Solving the supergravity equation of motion for D7 probe brane fluctuations in the two directions transverse to probe, $\left(\delta w_{5}=f(r) \sin (k \cdot x), \quad \delta w_{6}=\right.$ $h(r) \sin (k \cdot x))$ around the D7 brane probe embedding shown in Figure 3, the meson masses are given by $M^{2}=-k^{2}$. There are indeed two distinct mesons

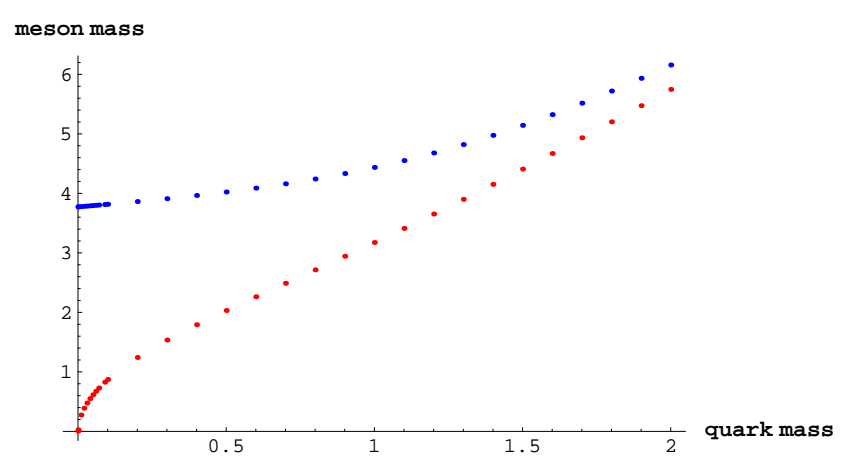

Figure 4: Meson mass versus quark mass as obtained from D7 probe brane fluctuations. The upper curve corresponds to the radial fluctuation, the bottom one to the $U(1)$ symmetric fluctuation. In units of $\Lambda_{\mathrm{QCD}}$. From [13]. (see Figure 4): One is massive for every $m$, and corresponds to fluctuations in the radial transverse direction, the other, corresponding to the $U(1)$ symmetric fluctuation, is massless for $m=0$ and is thus a Goldstone boson. It may be identified with the $\eta^{\prime}$, which becomes a $U(1)_{A}$ Goldstone boson for $N \rightarrow \infty$. At finite $N$, pure stringy corrections will give the $\eta^{\prime}$ a non-zero mass in the gravity picture, similarly to instantons in the field theory dual [15].

Another important property of the model of [13] is the small quark mass behaviour of the meson mass, proportional to the square root of $m$, thus satisfying the Gell-Mann-Oakes-Renner relation [16] of chiral QCD. Also the linear asymptotics for large $m$ correctly reproduce the field theory results. In [17] the R-scaling of the Goldstone's mass for small quark mass was determined as

$$
\frac{M_{\pi}^{2}}{\Lambda_{b}^{2}}=2.75 \sqrt{\frac{\pi}{g_{s} N}} \frac{m_{q}}{\Lambda_{b}} .
$$

The model has many remarkable QCD-like features. Note however that in the UV, it flows again to strongly coupled $\mathscr{N}=2$ theory with the degrees of freedom of $\mathscr{N}=4$ theory plus one additional fundamental hypermultiplet, and thus is not asymptotically free.

In figure 5 we plot the dependence of the rho meson mass on the pion mass squared in this model, in dimensionless units fixed by the choice of the supergravity scale $b$. The rho mass as function of the pion mass squared has recently also been computed for large $N$ within lattice gauge theory $[18,19]$, and a direct comparison of gauge/gravity and lattice results is possible. In the lattice computations, the scale is set by the value of the $\rho$ mass at vanishing $\pi$ mass. We choose our units such as to be able to compare directly with the lattice results of [19], which are shown on the right hand side of figure 5. In units such that the offset at $m_{\pi}=0$ coincides with [19], we find a linear dependence of the rho mass on the pion mass squared, with slope 0.57.

For the lattice results of [19], the simulations are performed in the quenched approximation. This is appropriate for the large $N$ limit, if not for smaller $N$. The lattice data of [19] is preliminary 

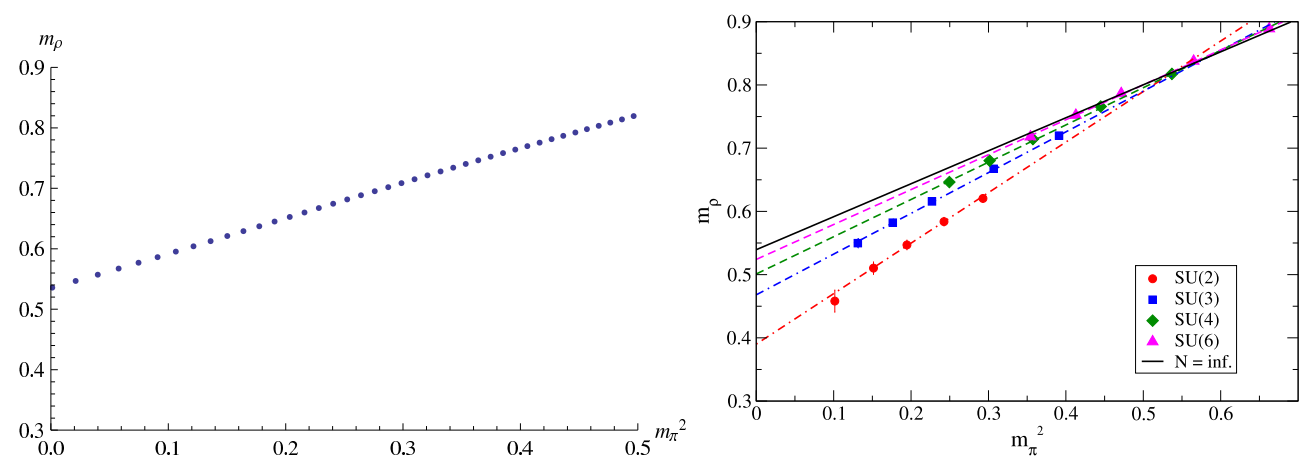

Figure 5: A plot of $m_{\rho}$ vs $m_{\pi}^{2}$ in the Constable-Myers background on the left. Lattice data [19] (quenched and at finite spacing) for the same quantity is also shown on the right.

and at a fixed, finite lattice spacing. Nevertheless it is striking that not only does the lattice data display the same linearity as the gauge/gravity model, but also the slope in the large $N$ limit is 0.52 and therefore is very close to the gauge/gravity dual result. The fact that the numbers agree at the level of the first digit is remarkable. The recent results of [20] show even better agreement.

\subsubsection{Sakai-Sugimoto model}

Sakai and Sugimoto [21] have considered a model of D4 branes with D8 and $\overline{\mathrm{D}} 8$ probes, with the 4-direction again compactified on a circle. The brane configuration in the probe limit is given by $N_{c}$ D4-branes compactified on a supersymmetrybreaking $S^{1}$ and $N_{f}$
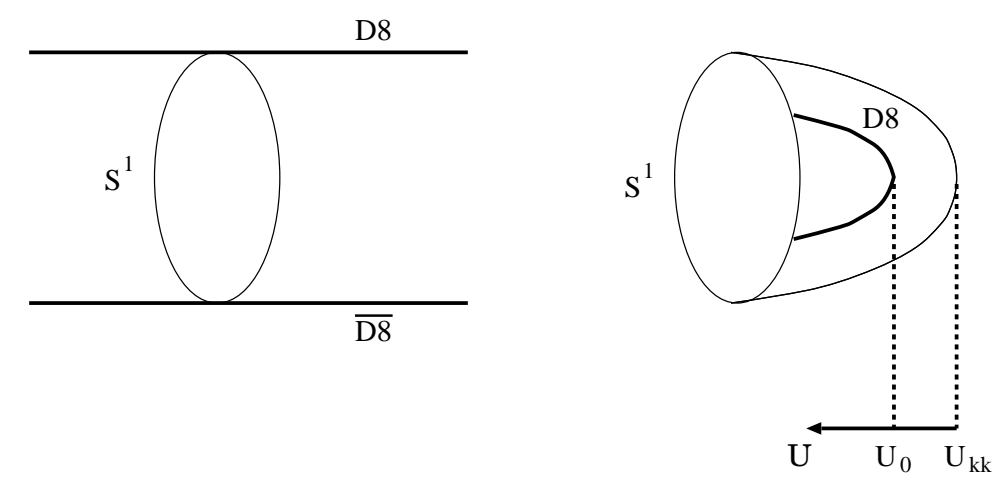

D8-D8 pairs transverse to this $S^{1}$. Anti-periodic boundary conditions are imposed for the fermions on the D4-branes in order to break SUSY and to cause unwanted fields to become massive. The $U\left(N_{f}\right)_{L} \times U\left(N_{f}\right)_{R}$ chiral symmetry in QCD is realized as the gauge symmetry of the $N_{f}$ D8-D8 pairs. The existence of the compact direction plays a crucial role in obtaining a holographic picture of chiral symmetry breaking. The radial coordinate $U$ transverse to the D4-branes is known to be bounded from below due to the existence of a horizon $U \geq U_{\mathrm{KK}}$ in the supergravity background, at which the radius of the $S^{1}$ shrinks to zero. It is found that the D8 and D8 branes merge at some point $U=U_{0}$ to form a single hypersurface (see Fig. 6), on which only a single factor of $U\left(N_{f}\right)$ survives. This mechanism is interpreted as the gravity dual of spontaneous breaking of $U\left(N_{f}\right)_{L} \times U\left(N_{f}\right)_{R}$ chiral symmetry.

This construction provides a model of $U(N)$ QCD with $N_{f}$ massless flavors. It allows for 
the calculation of a number of observables, such as meson masses, which may be compared with experiment. An example is the ratio of the $\rho$ and $a_{1}$ meson masses, for which Sakai and Sugimoto find $M_{\rho}{ }^{2} / M_{a_{1}}{ }^{2}=2.4$. The experimental value is 2.51 . The theoretical result is strikingly close, though of course it has to be stressed that it is hard to estimate the error of the prediction.

\section{References}

[1] J. M. Maldacena, Adv. Theor. Math. Phys. 2 (1998), 231 [Int. J. Theor. Phys. 38 (1999), 1113], [arXiv:hep-th/9711200].

[2] E. Witten, Adv. Theor. Math. Phys. 2 (1998) 253 [arXiv:hep-th/9802150]; S. S. Gubser, I. R. Klebanov and A. M. Polyakov, Phys. Lett. B 428 (1998) 105 [arXiv:hep-th/9802109].

[3] D. Z. Freedman, S. D. Mathur, A. Matusis and L. Rastelli, Nucl. Phys. B 546 (1999) 96 [arXiv:hep-th/9804058];

S. Lee, S. Minwalla, M. Rangamani and N. Seiberg, Adv. Theor. Math. Phys. 2 (1998) 697 [arXiv:hep-th/9806074];

M. Henningson and K. Skenderis, JHEP 9807 (1998) 023 [arXiv:hep-th/9806087].

[4] I. R. Klebanov and M. J. Strassler, JHEP 0008 (2000) 052 [arXiv:hep-th/0007191]; J. M. Maldacena and C. Nuñez, Phys. Rev. Lett. 86 (2001) 588 [arXiv:hep-th/0008001].

[5] J. Erdmenger, N. Evans, I. Kirsch and E. Threlfall, Eur. Phys. J. A 35 (2008), 81, [arXiv:0711.4467 [hep-th]].

[6] S. J. Brodsky and G. F. de Teramond, Phys. Rev. Lett. 96 (2006) 201601 [arXiv:hep-ph/0602252].

[7] J. Erlich, E. Katz, D. T. Son and M. A. Stephanov, Phys. Rev. Lett. 95 (2005) 261602 [arXiv:hep-ph/0501128].

[8] R. C. Brower, J. Polchinski, M. J. Strassler and C. I. Tan, arXiv:hep-th/0603115.

[9] L. F. Alday and J. Maldacena, JHEP 0706 (2007) 064 [arXiv:0705.0303 [hep-th]].

[10] A. Karch and E. Katz, JHEP 0206 (2002), 043, [arXiv:hep-th/0205236].

[11] P. Breitenlohner and D. Z. Freedman, Phys. Lett. B 115 (1982) 197.

[12] M. Kruczenski, D. Mateos, R. C. Myers, and D. J. Winters, JHEP 07 (2003), 049, arXiv:hep-th/0304032.

[13] J. Babington, J. Erdmenger, N. J. Evans, Z. Guralnik and I. Kirsch, Phys. Rev. D 69 (2004), 066007, [arXiv:hep-th/0306018].

[14] N. R. Constable and R. C. Myers, JHEP 11 (1999), 020, arXiv:hep-th/9905081.

[15] J. L. F. Barbon, C. Hoyos, D. Mateos, and R. C. Myers, JHEP 10 (2004), 029, arXiv:hep-th/0404260.

[16] M. Gell-Mann, R. J. Oakes, and B. Renner, Phys. Rev. 175 (1968), 2195.

[17] N. J. Evans and J. P. Shock, Phys. Rev.D70 (2004), 046002, arXiv:hep-th/0403279.

[18] G. Bali and F. Bursa, PoS LATTICE2007 (2006), 050, [arXiv:0708.3427 [hep-lat]].

[19] L. Del Debbio, B. Lucini, A. Patella and C. Pica, JHEP 0803 (2008), 062, [arXiv:0712.3036 [hep-th]].

[20] G. S. Bali and F. Bursa, JHEP 0809 (2008) 110 [arXiv:0806.2278 [hep-lat]].

[21] T. Sakai and S. Sugimoto, Prog. Theor. Phys. 113 (2005), 843, arXiv:hep-th/0412141; T. Sakai and S. Sugimoto, Prog. Theor. Phys. 114 (2006), 1083, arXiv:hep-th/0507073. 